EOMmUn: Communication et organisation

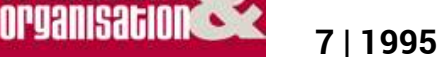

L'entreprise et ses mémoires

\title{
La tradition du futur
}

Nicole Denoit

\section{OpenEdition}

Journals

Édition électronique

URL : http://journals.openedition.org/communicationorganisation/1781

DOI : 10.4000/communicationorganisation. 1781

ISSN : 1775-3546

\section{Éditeur}

Presses universitaires de Bordeaux

Édition imprimée

Date de publication : 1 mai 1995

ISSN : 1168-5549

\section{Référence électronique}

Nicole Denoit, "La tradition du futur », Communication et organisation [En ligne], 7 | 1995, mis en ligne le 26 mars 2012, consulté le 19 avril 2019. URL : http://journals.openedition.org/

communicationorganisation/1781; DOI : 10.4000/communicationorganisation.1781

Ce document a été généré automatiquement le 19 avril 2019

(c) Presses universitaires de Bordeaux 


\title{
La tradition du futur
}

\author{
Nicole Denoit
}

\section{NOTE DE L'ÉDITEUR}

Le titre de cet article est celui que Jean-Michel FOLON a choisi pour son tableau original de 1985, commandé par la société LU/BSN pour l'enrichissement de son Patrimoine Artistique Contemporain.

\section{Le Petit-Beurre Lu : Patrimoine national}

1 Lorsqu'il y a quelques années le journal MARIE CLAIRE enquêtait sur les « 99 raisons de se réjouir d'être français» le Petit-Beurre arrivait en tête du classement de nos compatriotes, largement devant d'autres éléments mythiques de notre patrimoine national comme le camembert, le bifteck frites ou la $2 \mathrm{CV}$. Le Petit-Beurre serait donc classé patrimoine national.

2 Étonnante aventure en effet d'une mémoire collective, plus jalouse parfois que l'entreprise elle-même, de l'image qu'au fil des générations de LEFEVRE-UTILE elle a su créer à travers un produit local, dont la valeur est aujourd'hui plus mythique que commerciale ou plus exactement commerciale parce que mythique.

On mange beaucoup moins de Petit-Beurre et plus généralement le marché des biscuits secs ne représente qu'une part réduite du marché global, soit $20,2 \%$ en 1992, part lourdement menacée par les nouveaux biscuits chocolatés.

Cependant, une étude effectuée par BSN en 1988 auprès des consommateurs montre que le public interrogé sur la marque LU l'associe immédiatement et prioritairement au petitbeurre nantais. Moins goûté, il continue à exercer sa toute puissance sur la mémoire collective. 
Qu'en est-il du côté de l'entreprise nantaise qui fêtait en 1986 le centenaire du PetitBeurre LU ? Car c'est bien à Nantes qu'est né, le 8 septembre 1886, du génie de Louis Lefèvre-Utile, le fameux « Petit-Beurre LU-Nantes ».

\section{Un lieu de mémoire à la dérive : La frustation des nantais}

6 Les derniers feux des «Allumés » de Nantes éclairent curieusement, en septembre 1994, cette remontée dans la mémoire de l'usine LU : la grande carcasse métallique abandonnée sur l'ancien quai de la Loire, le quai Ferdinand Favre, en plein centre ville, offrant aux Nantais «deux morceaux défaits et pathétiques ${ }^{1}$ ", vestiges d'une gloire déchue, donne pour quelques soirs l'hospitalité aux musiciens du Caire, ville invitée pour une semaine, selon la tradition des "Allumés », manifestation culturelle d'envergure proposée depuis quelques années par la ville de Nantes.

7 La mairie de Nantes, propriétaire depuis 1991 des anciens bâtiments de l'usine LU, ouvre l'espace, redonnant au lieu une étrange raison d'être, une résurrection aussi inattendue qu'éphémère, un « détournement de mémoire ».

8 L'odeur des produits orientaux remplace celle du Petit-Beurre mais les Nantais sont heureux de réinvestir ce lieu mythique, pan d'une histoire locale, d'une mémoire, pas seulement celle de l'entreprise mais de leur ville, de leur enfance pour certains.

De cet attachement, Michel Ragon parle avec nostalgie dans une page du catalogue de l'exposition «L'ART ET LES BISCUITS - LE PATRIMOINE ARTISTIQUE CONTEMPORAIN LU », proposée à l'occasion du centenaire du Petit LU en 1986.

«Pour qui a vécu sa jeunesse à Nantes, ce qui est mon cas, LU fait partie de la mythologie familiale. Le Pont Transbordeur, le Passage Pommeraye et la Biscuiterie LU ont été les trois merveilles de mon adolescence. Ces trois mots, si souvent déchiffrés sur des affiches, des réclames : LU-PETIT BEURRE-NANTES, sont pour moi indissociables [...] L'usine Lefèvre-Utile se trouvait quai Baco. Car il y avait un quai, en ce temps-là, la Loire longeant l'édifice flanqué de deux tours à coupoles (hélas décapitées) et de hautes cheminées fumantes [...] Le petit garçon à pèlerine, à béret et à bottines, arborant sa croix d'honneur et son biscuit LU, c'est moi. Absolument. Personne d'autre.»

10 Par contraste, l'étonnement saisit le visiteur de passage qui découvre aujourd'hui, en plein centre ville et à l'abandon, les bâtiments tronqués, la tour décapitée qui constituent «tout ce qui demeure des Établissements Lefèvre-Utile qui furent un peu la gloire de la ville. $»^{2}$

11 Des rumeurs animent les conversations. Les Nantais rêvent et rapportent des projets ambitieux, contradictoires, de réhabilitation de l'ancienne usine LU. Tous se sentent concernés.

12 Actuellement, les bâtiments tombent en ruine: réduits, mutilés progressivement et délibérément au cours des ans, enfin abandonnés puis vendus sans état d'âme par le géant BSN qui devait absorber LU en 1986.

13 Longtemps, du niveau supérieur des deux tours qui formaient la porte de l'avenue Carnot, les trompettes de la Renommée, autrefois symbole de la marque, ont rayonné sur la ville entourée de l'universalité des signes du zodiaque. Seule la tour de gauche est aujourd'hui maintenue mais la trompette de la Renommée a disparu... Triste symbole d'une 
dégradation progressive, d'un abandon dont souffrirent les ouvriers, déplacés depuis 1986, pour cause de modernisation, dans la nouvelle usine de La Haie Fouassière à $14 \mathrm{Km}$ de Nantes.

Pourquoi cet exil décidé par le PDG de GENERALE BISCUIT, Claude-Noël Martin ?

« Nous voulions une usine à la campagne. Nous avons été séduits par le côté rural,

traditionnel. C'est ainsi que les étrangers imaginent la France.»

Volonté donc de s'inscrire dans une mémoire collective, celle d'une France rurale, de se détacher désormais de la mémoire $L U$, du patrimoine industriel et familial d'une époque glorieuse. Une autre mémoire pour d'autres temps.

De ce lieu de mémoire à la dérive, on peut reconstituer les étapes d'édification, d'une architecture certes, mais surtout d'une symbolique habilement construite, d'un imaginaire fortement attaché au site en plein cœur de la ville.

\section{6-1909 : Une mémoire se construit, un patrimoine culturel s'édifie}

Lorsque Jean Romain Lefèvre, issu d'une famille de l'Est de la France, s'installe en 1846 à Nantes, il apporte déjà avec lui, à 27 ans, une mémoire, celle d'une tradition, d'un savoirfaire : le biscuit rose de Reims qu'il fabrique et vend à la modeste pâtisserie de la rue Boileau.

Cette mémoire, avant d'être celle de l'entreprise, est celle d'un artisanat de qualité, celle d'un métier exigeant dont on apprend respectueusement les traditions. Son mariage avec Pauline-Isabelle Utile en 1850 donnera son nom à l'entreprise désormais familiale : la maison « LEFEVRE-UTILE ».

La pâtisserie de la rue Boileau, qui avait abrité la génération des fondateurs, étant devenue trop petite, la construction de l'usine va commencer en 1885, avec Louis LefèvreUtile, le long de la Loire dans l'ancienne filature Bureau. L'entreprise passe subitement de 150 à $2000 \mathrm{~m} 2$, parallèlement à une modernisation considérable des systèmes de production. L'entreprise familiale artisanale s'industrialise. Le Petit-Beurre naîtra un an plus tard dans ces nouveaux locaux.

21 Ces quelques éléments témoignent d'une réussite industrielle spectaculaire mais ne nous intéressent que dans la mesure où ils sont associés à la lucidité et à la volonté de Louis Lefèvre-Utile d'édifier un véritable patrimoine architectural et culturel pour son entreprise, une mémoire pour l'avenir en même temps qu'une mémoire pour la ville.

Ainsi, dès 1888, Louis Lefèvre-Utile avait compris l'importance de cette façade d'usine en face de la gare et du château des Ducs: tradition et modernité seront les valeurs porteuses de l'aventure LU et restent encore aujourd'hui la ligne directrice de la politique de communication.

Louis Lefèvre-Utile décide d'embellir l'ancienne filature et d'aménager un grand portail monumental en plein cintre. Il transforme le dernier étage carré en immense bandeau en attique avec l'inscription «Manufacture Biscuits Lefèvre-Utile». Tout concourt à la monumentalisation de l'espace de production tandis qu'à l'ouest, la façade néo-classique des bureaux assure aux clients l'image cossue de la réussite. 
24 Le génie de Louis Lefèvre-Utile est d'avoir lié intimement l'expansion des ateliers de fabrication à l'image de marque de l'usine dans la ville. La mémoire de l'entreprise s'inscrit définitivement dans la mémoire collective des Nantais.

Au tournant du siècle, la grande affaire de l'usine est la construction de l'ensemble de la façade de chaque côté de la nouvelle avenue Carnot; Louis Lefèvre-Utile explicite clairement auprès de la mairie, pour négocier des échanges de terrains, sa volonté de magnifier l'entreprise LU, conscient de ce que le prestige de l'entreprise pouvait apporter à l'image de la ville.

On ne parlait pas encore de politique de communication des villes mais c'est bien de cela dont il s'agit dans cette transaction qui s'est opérée entre Louis Lefèvre-Utile et la mairie. Le compte rendu du conseil municipal du 27 décembre 1899 montre que la ville est consciente du retour d'image qu'entraînera pour elle la politique architecturale de Louis Lefèvre-Utile.

28 "Afin d'augmenter l'étendue de son usine et de lui donner un meilleur aspect, la société Lefèvre-Utile souhaite devenir propriétaire des deux côtés de l'avenue. Elle se propose d'édifier de chaque côté de l'avenue Carnot des constructions symétriques d'aspect monumental. Ces constructions seront de belle apparence et donneront à l'entrée de l'avenue Carnot un aspect des plus agréables. »

29 Les tours LU, réalisées par l'architecte Auguste Blyssen, sont construites en 1903. Elles représentent pleinement l'architecture de la Belle Époque de par leur composition qui allie Art Nouveau, tradition et modernité. Le sigle LU, l'Ange de la Renommée qui les couronne, chargé d'annoncer la suprématie de la qualité de la maison, les décors des coupoles, les vitraux et mosaïques témoignent du goût de la Belle Époque.

30 À cet art s'allie la nouvelle architecture industrielle, les coupoles en armature métallique, l'utilisation des premiers bétons armés.

31 Les tours s'illuminent le soir et s'élèvent comme l'emblème de la prospérité industrielle de la famille, de la ville, de la région.

32 Les tours LU sont imaginées comme la réplique, à l'ère industrielle, des tours du château qui, comme elles entourées d'eau, leur font face. Postées à l'angle comme des sentinelles, elles veillent sur l'enfilade des bâtiments. Ces portes d'une immense biscuiterie transforment une usine en château de l'industrie.

33 L'architecture de l'usine LU est conçue pour s'inscrire dans la mémoire de la ville, dans un patrimoine édifié au cours des âges. Les Nantais s'y reconnaîtront de ce fait tout naturellement.

34 Cette double volonté d'intégration et de distinction dans un patrimoine collectif est sans doute un des éléments essentiels de la pérennité étonnante de la " Mémoire LU », malgré les erreurs de gestion qui suivront.

La politique de communication de Louis Lefèvre Utile - sa lucidité et la cohérence de ses démarches innovantes nous permettent d'employer cette expression- intègre, dès sa mise en place, la notion de " mémoire d'entreprise ".

Louis Lefèvre-Utile, lui-même passionné de photographie, va "mettre en scène » son usine, photographiant en 1900 l'ensemble des employés de l'usine LU dans le grand hall d'expédition du quai Baco. Dans la constitution de cette mémoire photographique, tous les détails de la mise en scène sont prévus : «Il sera nécessaire de pourvoir quelques ouvriers et ouvrières, de préférence minces et bien faits, de tabliers, blouses, pantalons et 
bonnets blancs. Soigner aussi les chaussures, bas et sabots. Ce personnel choisi animera les vues où il est nécessaire d'avoir du mouvement, des gestes et de l'animation... $»^{3}$ Tels sont les propos du fils, Michel Lefèvre-Utile préparant les photographies de l'usine prises pour l'exposition internationale de 1937. Louis Lefèvre-Utile avait aussi parfaitement compris l'importance de la vue aérienne pour la précision des images publicitaires. La première fut réalisée par un cliché pris d'un ballon aux couleurs de Lefèvre-Utile lancé à l'occasion du 14 juillet 1898. Ici encore se superposent habilement la mémoire de l'entreprise et la glorieuse mémoire collective.

La mémoire de l'entreprise LU s'est édifiée, on l'a compris, à l'écoute de la mémoire collective, du patrimoine architectural de la ville mais aussi plus largement des événements marquants du monde contemporain. Ainsi, les grands événements de l'actualité sont systématiquement exploités par Louis Lefèvre-Utile pour en faire des cartes chromolithographiques de petite taille, placées dans les paquets de biscuits. Pour chaque thème, une série de cartes différentes étaient établies afin d'inciter le client à les collectionner. De nombreuses séries ont été crées à partir de grands événements.

Faire acheter, certes, mais aussi rattacher le prestige de l'entreprise aux grandes heures de son temps, tel est le projet de Louis Lefèvre-Utile. Susciter le désir de collectionner c'est bien sûr fidéliser la clientèle mais aussi l'inviter à se souvenir, l'attacher à une mémoire qui n'est pas seulement celle de l'entreprise mais d'une époque toute entière.

Le premier grand thème fétiche est l'horizon lointain, les terres nouvelles découvertes par le colonialisme. Une série de cartes représente la conquête de l'Afrique, avec des paysages de végétation sauvage et des petits enfants au costume traditionnel sur la plage. Les cartes autographes des albums de LU représentent des pays nouveaux comme Madagascar, avec l'autographe de sa reine et un décor exotique, d'autres montrent des scènes de la colonisation ou encore une scène de l'évangélisation de la Martinique.

Certaines cartes valorisent les progrès techniques de l'époque. Une série rapporte les exploits océanographiques du Commandant Charcot, un biscuit est baptisé Caravelle, une série de cartes postales illustre les exploits des grands aviateurs du moment comme Louis Blériot.

Enfin, l'un des thèmes abordés par les cartes publicitaires LU à collectionner sont les fêtes franco-russes à l'occasion de la visite du Tsar à Paris pour sceller l'accord diplomatique entre les deux pays, accord ratifié le 27 décembre 1893. Après cet engouement pour l'alliance franco-russe, Louis Lefèvre-Utile crée le Biscuit Russe Néva.

Toutes ces créations relèvent d'une démarche publicitaire mais qui s'opère dans un souci de mémoriser, de dater, de fixer les étapes d'une longue vie pour l'entreprise. Mémoriser, c'est manifester sa fierté du présent mais aussi sa confiance en l'avenir.

43 La volonté de Louis Lefèvre-Utile de rattacher l'image de son entreprise à un patrimoine collectif s'affirme aussi dans les liens qu'il établit avec les artistes talentueux de sa génération: Cappiello, Luigi Loir, Benjamin Rabier, Mucha dont les nombreuses œuvres réalisées pour LU constituent une mémoire exceptionnelle de l'entreprise mais aussi d'une époque dans ce qu'elle a de plus brillant.

Aux « célébrités contemporaines » Louis Lefèvre-Utile fait vanter les mérites des biscuits LU et publie en un album la présentation de vingt-huit personnalités qui ont accepté de parler du Petit LU. Ainsi Sarah Bernhardt déclame-t-elle : «Je ne trouve rien de meilleur qu'un Petit-LU. Oh, si ! Deux Petits-LU !» 

faire, celle d'une histoire familiale mais aussi régionale et nationale qui installe la société LU dans une tradition d'exigence et de réussite. Associer la marque LU aux grands événements de l'histoire, c'est lui donner une mémoire collective, une existence qui s'imposera dans la durée comme une évidence, dans une sorte de "tradition du futur » selon le mot de Jean-Michel Folon.

\section{0-1978 : Une mémoire encombrante}

Cette mémoire LU, d'une extraordinaire diversité et en même temps d'une grande cohérence, attachée à la seule personnalité de Louis Lefèvre-Utile va devenir un élément de résistance à la modernisation de l'entreprise. Mémoire « encombrante » donc, jusqu'en 1943, pour la génération suivante des Lefèvre-Utile.

Mémoire " encombrante » aussi pour ceux, qui, avides de pouvoir, ne peuvent supporter ce passé, cette mémoire, dont ils sont exclus. Mémoire menacée donc, lorsque la société LU passera en 1968 aux mains de la direction BRUN.

Mémoire «encombrante » enfin, lorsqu'elle impose en 1974, à la direction LU-BRUN et Associés, par sa présence architecturale problématique, des préoccupations qui ne sont pas encore à l'ordre du jour et s'oppose ainsi aux spéculations immobilières du moment. Commencera alors une destruction progressive du patrimoine architectural LU.

Revenons sur ces trois étapes de contestation puis de destruction de la mémoire LU.

L'entreprise LU va donc, avec une inventivité prodigieuse jusqu'à la guerre de 1914-1018, manifester sa renommée, en l'inscrivant dans une tradition qu'elle s'est construite, mais qui va peu à peu la brider, l'enfermer pour quelques années dans une attitude passéiste. Après quarante ans d'ascension fulgurante, la situation va se dégrader. L'entre-deux guerres est une période de stabilité, voire de stagnation. Louis Lefèvre-Utile s'est en effet crispé sur les techniques de 1900, fidèle à cet esprit de qualité qui lui fait rejeter toute mécanisation supplémentaire.

51 Il transmettra ses craintes aux ouvriers qui, formés au respect des traditions, accepteront avec méfiance en 1951 (alors que le concurrent BN est équipé depuis 1939) les premiers essais d'une ligne de fabrication continue où laminage, découpe et cuisson se déroulent sans interruption. Dans les années 30, c'est donc l'immobilisme total. La gamme des produits, la publicité, le matériel restent les mêmes alors qu'autour de Louis Lefèvre-Utile tout change. La société LU commence à ressembler à une usine semi-artisanale dont les méthodes, les machines, ne sont plus adaptées.

Son fils Michel essaye de convaincre son père, mais il ne veut rien entendre. Il dirige tout en patriarche, d'une main de fer

53 Louis Lefèvre-Utile a voulu et su construire pour son entreprise une image de marque, de tradition, une mémoire, mais le problème se pose ensuite de rester réceptif à la modernité. Les traditions, les souvenirs sont riches d'enseignement et de sagesse mais on sait qu'ils apparaissent comme signe de vieillissement lorsqu'ils tiennent lieu de tout.

Louis Lefèvre-Utile ne supportera pas l'occupation de son usine par les Allemands et mourra en 1940.

55

On entre alors, après la guerre, dans une phase de modernisation. Michel et Patrick Lefèvre-Utile, longtemps assujettis au pouvoir du patriarche, se dégagent des traditions 
familiales et se tournent résolument vers ce qui incarne la modernité : les Etats-Unis. Patrick Lefèvre-Utile part en 1947 pour se former aux nouvelles techniques du design, inventé par l'ingénieur parisien installé à New-York, Raymond Loewy. Après y avoir appris les notions de conditionnement et de publicité, Patrick Lefèvre-Utile revient en France, persuadé de la nécessité de repenser l'usine LU.

C'est d'abord en 1951 l'installation d'une ligne continue permettant la production de masse des Petits-Beurre. Les années cinquante marquent donc une étape de rajeunissement. L'entreprise se réfère peu à sa propre mémoire et on abandonne le souci d'inscrire l'entreprise dans une mémoire collective, un patrimoine culturel. Cette attitude correspond à la mentalité du moment qui remet en cause les valeurs traditionnelles. Chacun invente ses propres points de repère, l'entreprise doit en faire de même.

Fidélité cependant à une vieille tradition de la famille LU : faire appel aux plus grands. Patrick Lefèvre-Utile s'adresse en 1957 au plus grand designer de l'époque, Raymond Loewy, celui qui a imposé le sigle Coca-Cola, et lui demande un nouveau logo : ce sera LU tel qu'on le retrouve encore aujourd'hui.

Cette pérennité du sigle est événement déterminant de la mémoire de LU.

Un travail sur le logo et l'emballage s'imposait en fonction des nouvelles structures de distribution: les premières supérettes, où la lisibilité des marques est un facteur déterminant de la vente en libre-service. On est alors loin de la clientèle bourgeoise et privilégiée à laquelle était destinée la production LU.

En ces années-là, il n'est donc pas de mode de jouer sur la tradition et la mémoire de l'entreprise LU. Ainsi, après un nouvel habillage de neuf, grâce à Loewy, l'année 1957 voit se dessiner une campagne Petit-Beurre de sensibilisation. Celle-ci joue sur la rupture avec l'image traditionnelle du Petit-Beurre. En effet, Hunguerer, grand dessinateur de publicité, en fait le partenaire d'animaux inattendus. On joue sur l'effet de surprise, d'insolite mais d'autant plus efficace peut-être, qu'il s'oppose à la longue tradition LU installée dans l'imaginaire collectif.

61 Quant au prestige du lieu, il a souffert: les bombardements ont touché l'usine, les tours sont fragilisées. Il semble qu'on ait pensé les détruire pour des raisons d'alignement. Un projet de reconstruction des dômes est demandé plus tard à Raymond Loewy, vers 1960. Il n'aura pas suite.

62 En 1967, devant la multiplication des biscuiteras et leur concurrence stérile, Patrick Lefèvre-Utile propose de mener une politique d'alliances sur le plan national pour mieux percer à l'exportation. Ainsi, en avril 1968, LU s'associe avec cinq fabricants : BRUN, TROIS CHAYONS, St SAVEUR, REM, MAGDELEINE. Ils fondent ainsi le groupe LU-BRUN et Associés. Patrick Lefèvre-Utile est nommé Directeur Général de la nouvelle société. En 1960, Patrick Lefèvre-Utile est évincé du groupe. André Forgeot, patron de BRUN prend son fauteuil de président.

63 Commence alors pour la mémoire LU, le patrimoine de la société, une destruction délibérée, liée à la volonté de pouvoir de la société BRUN.

Le siège social est immédiatement transféré de Nantes à Paris malgré les protestations des employés et des syndicats. La direction parle de concentrer ses fabrications à Reims dans une nouvelle usine dotée des derniers perfectionnements techniques. Le fameux Petit-Beurre associé depuis 1886 à l'image de la ville ne serait plus nantais. Les manifestations de protestation sont nombreuses, les Nantais sont bouleversés par le mépris de leur patrimoine. Le projet ne se réalisera pas pour des raisons de subvention. 
L'usine revient de loin ; mais son patrimoine va subir des affronts plus secrets.

Un témoin de l'époque, responsable des archives et employé dans les bureaux du quai Baco, raconte comment les archives de la société LU, manuscrits, livres de compte divers, sont jetés par camions entiers sur ordre de la nouvelle direction.

Le service des Archives Départementales sera alerté par ce témoin, fidèle à l'entreprise LU pendant 35 ans et soucieux de sa mémoire. Une partie des documents sera sauvée.

Il est difficile d'analyser cette période de réorganisation, pour le moins brutale, des services de gestion par la nouvelle direction. On retiendra cependant l'attachement du personnel à la mémoire de l'entreprise et au sentiment d'une dépossession illégale qui les révolte et contre laquelle il luttera souvent individuellement. Soit en intervenant auprès des organismes officiels qui pouvaient l'entendre: Archives Départementales, Musée, Service Régional de l'Inventaire, soit en rassemblant individuellement quelques éléments voués à la destruction.

9 Les traces de la mémoire LU, devenues peut-être inutiles à la nouvelle gestion, faisaient obstacle à sa volonté de puissance. Une mémoire, même inopérante, gêne par sa seule existence. On sait avec quel plaisir et pourquoi on brûle les cahiers qui ne servent plus. La démarche peut être aussi simpliste et naturelle. Elle devient dangereuse et contestable lorsqu'elle concerne la collectivité.

Voici donc une partie du patrimoine éparpillée, mais en même temps répertoriée, classée, par ceux qui en ont hérité. En sortant de ses murs, la mémoire de l'entreprise fructifie.

L'abandon, pire la volonté destructrice de l'entreprise qui a changé de mains, va entamer un processus de valorisation d'une mémoire passée dans le domaine public.

2 En 1974, les grands bureaux aménagés en 1897 quai Baco doivent être détruits pour répondre à des spéculations immobilières. Il faut alors déménager en toute hâte ce qui peut être sauvé du patrimoine qui est ici accumulé et dont, souvent, on ne sait pas la valeur, notamment celle des objets publicitaires de toutes sortes que la génération de Louis Lefèvre-Utile avait produits.

Dans ces moments-là, une quantité extraordinaire de ces objets fut éparpillée, conduite à la décharge, récupérée innocemment par ceux qui y retrouvaient leur passé, moins innocemment par des antiquaires avertis. On peut dire qu'un véritable commerce s'est établi dans les circonstances anarchiques du déménagement. Cet éparpillement du patrimoine LU, par le même phénomène qui fait monter la côte d'un artiste lorsque ses œuvre se répandent, va contribuer à valoriser toutes les traces de la mémoire de LU, leur conférant une véritable valeur marchande.

4 La famille Lefèvre-Utile, et en particulier Michel Lefèvre-Utile, écarté de la direction, se sentent désormais désarmés face à l'important patrimoine qu'ils doivent sauver dans les conditions difficiles de l'urgence. Ils font alors appel à Jacques Cailleteau, Conservateur Régional de l'Inventaire Général et lui remettent «un ensemble extraordinaire de documents iconographiques $»^{4}$, toute une série de plaques photographiques qui permettront, bien plus tard, en 1989 au Service de l'Inventaire de publier un ouvrage sur l'usine LU dans la collection « Images du Patrimoine ».

75 Ici encore, de cet éparpillement douloureux pour l'entreprise et ceux qui lui sont attachés, naîtra une valorisation exceptionnelle du patrimoine, par les spécialistes qui ont recueilli les documents voués à la destruction. 


\section{8-1986 : Une mémoire à reconstruire} donc courir les antiquaires et les ventes de la collection LU, éparpillées au gré des mutations économiques précédemment évoquées. L'entreprise rachète peu à peu ce qui lui appartenait autrefois. Une reconstruction patiente et déterminée commence, étonnante démarche qui prendra plusieurs années, à laquelle on consacrera temps et argent. L'entreprise, dépossédée de sa mémoire, part à la recherche d'elle-même. contemporains a pour but de "développer l'image institutionnelle de $\mathrm{LU}$ », tandis que 
l'intention initiale de LU, dans cette collection constituée au fil des années, était uniquement publicitaire.

Les œuvres anciennes et contemporaines sont présentées annuellement dans divers pays $\mathrm{du}$ monde. Une mémoire transportable donc, véritable "mallette pédagogique» au service de l'image de l'entreprise. Une mémoire sans lieu, une culture exportable.

GENERALE BISCUIT avec Claude-Noël Marin jouent la carte de la mémoire d'entreprise, suivant en cela la tendance du moment vers le mécénat et la constitution de collections. Cependant, si la mémoire LU est reconstruction, les bâtiments de l'usine nantaise subissent des destructions. Ainsi, en 1977, la cheminée LU, haute de $35 \mathrm{~m}$ est détruite. "Pour les uns, elle était une verrue inesthétique, pour les autres un témoin d'un passé qui méritait quelques souvenirs... » C'était en quelque sorte le dernier acte de la démolition des bâtiments Lefèvre-Utile, allée Baco. Pour LU-BRUN elle représentait 8 à 900 tonnes de ferrailles... et pour les Nantais, une foule de bons souvenirs... $\|^{5}$

C'est sous la direction de Claude-Noël Martin qu'est décidée la construction de la nouvelle usine de la Haie Fouassière. Patrick Lefèvre-Utile, lui-même, ne voit dans cette décision que la volonté d'abandonner des bâtiments trop anciens et un matériel obsolète. La direction ne se désintéresse pas de l'avenir du lieu qui va être abandonné. Dans l'esprit de la collection « Patrimoine LU » que l'entreprise a constituée, Claude-Noël Martin imagine de réaliser dans les anciens locaux un musée du biscuit où une vingtaine d'ouvriers, en costume d'époque, feraient fonctionner pour les spectateurs une chaîne de Pailles d'Or. Le maire de Nantes, Monsieur Chauty, réagit très mal à cette proposition. «Plaisanterie lamentable! Et pourquoi pas un musée de la construction navale chez Dubigeon et un musée de la conserverie chez Cassegrain? Nantes serait la ville-musée la mieux organisée de France $»^{6}$

Claude-Noël Martin n'aura pas le loisir de s'opposer davantage au maire de Nantes. Il sera absorbé par le géant BSN sans préavis en 1986. L'usine déménagera comme prévu à la Haie Fouassière et les anciens bâtiments resteront à l'abandon.

BSN négociera pour vendre à prix fort l'usine de Nantes à la mairie. La vente n'aura lieu qu'en juillet 91.

91 Commercialement, la construction d'une nouvelle usine performante, informatisée, s'explique. Mais on ne se débarrasse pas aussi facilement et inpunément d'un lourd passé local. La mémoire résiste, se refuse à l'amnésie qu'on préconise.

Nantes toute entière aujourd'hui se souvient, proteste, et la politique de communication de la ville intègre comme une nécessité incontournable la demande de réhabilitation de ce lieu de la mémoire qu'est l'usine LU quai Ferdinand Favre. La réalisation est toujours pour demain... En attendant l'usine est toujours davantage réduite. En 93, on en démolissait encore toute une partie pour réaliser une ensemble immobilier.

Aujourd'hui également, à la Haie Fouassière, on écoute davantage les ouvriers, politique de communication oblige. On a récemment installé dans le hall d'entrée, irréprochable de modernisme, un ancien pétrin qui propose au regard nostalgique de l'ouvrier ou curieux du visiteur, un signe de respect pour le passé, le "savoir-faire » des anciens. Mais le perfectionnisme de la présentation, le vernis, - non-sens technique-, qui enferme l'objet dans une gangue lisse, fait de lui un pauvre souvenir emballé, figé, oublieux de ce que fut son fonctionnement. Comble d'ignorance: ce pétrin viendrait non pas de l'usine de Nantes, mais de l'usine de Grenoble, usine BRUN, usine rivale ! 

lire la dépossession d'une usine privée de son lieu et de sa mémoire? Le trouble de l'observateur augmente face à cette situation lorsqu'il apprend l'existence d'un dossier publié par le Service de l'Inventaire général en 1989, intitulé «L'Usine LU»; dossier que la société BSN n'aurait acheté qu'en quatre ou cinq exemplaires au prix modique de 90FF... Héritage divisé, dont les différents dépositaires s'ignorent. Il semble bien que le groupe BSN poursuive une politique de communication qui intègre la mémoire de LU sous sa forme transportable, modulable, mais désormais coupée de son lieu de mémoire, de ses racines géographiques.

passage d'une mémoire d'un lieu à une mémoire sans lieu manifeste bien tout l'écart de sensibilité qu'il peut y avoir entre une entreprise familiale et un géant mondial. Seule la qualité artistique de cette mémoire la rend intégrable, exploitable dans un contexte commercial international.

\section{BIBLIOGRAPHIE}

Biscuits LU- Groupe BSN Le patrimoine artistique LU de 1880 à nos jours. Paris 1990

Catalogue de l'exposition au Château Des Ducs De Bretagne. Biscuiteries de Nantes du XIX ${ }^{e}$ siècle à nos jours. 1987

GUERIN S. La saga du Petit-Beurre LU de 1886 à 1993.

Mémoire de maîtrise d'Histoire économique, Université Paris I Sorbonne septembre 1993.

HERSCHER G. L'art et les biscuits, publicité de la firme Lefêvre-Utile de 1897 à 1914. Chêne, Paris 1978.

Communication et organisation, 7 | 2012 
Inventaire Général Des Monuments et des richesses artistiques : Images du patrimoine de l'Usine LU $n^{\circ} 59$

Périodique : ARMEN La Bretagne, un monde à découvrir. Biscuits $L U$ : un siècle d'industrie gourmande. $\mathrm{n}$

- 11, octobre 1987 p. 2 à 33

\section{NOTES}

1. Revue 303 Pays de Loire

2. Revue 303 Pays de la Loire

3. Image du Patrimoine $\mathrm{N}^{\circ} 59 \mathrm{LU}$. Une usine à Nantes $\mathrm{p} 1$

4. Ouest-France du 30/04/77

5. Ouest-France du 30/04/77

6. La Tribune $\mathrm{n}^{\circ} 182$ du 19 au 25 septembre 1985

7. Journal de l'usine LU de la Haye Fouassière

\section{RÉSUMÉS}

Nous proposons ici à l'observation l'aventure originale d'une mémoire d'entreprise devenue patrimoine national, celle de la biscuiterie nantaise LU, aujourd'hui absorbée par le groupe BSN.

De la mémoire d'un lieu à une mémoire sans lieu, de l'entreprise familiale au géant BSN, un héritage éclaté, à plusieurs reprises menacé, mais d'une exceptionnelle richesse, dont nous étudierons les différentes stratégies de destruction ou d'appropriation.

The purpose of this study is to present the original destiny of the "memory of the compagny " the LU Biscuit factory based in Nantes, a part of a national heritage which has been absorbed by BSN conglomarate. This resurche will show the transition of a place with a history turned into a history without place: it will examine the transformation of this familiy entreprise of exceptionnal interest taken over the BSN geant, whose cultural patrimony was scattered away and several times threatened in its very existence, and it will concentrated on a various strategies implemented either to destroy or control

\section{AUTEUR}

\section{NICOLE DENOIT}

L'auteur est chargée de cours à l'Université du Maine en « Techniques d'expression et de communication », au Département de Langues Etrangères Appliquées au Commerce International. Docteur ès Lettres, elle s'intéresse actuellement à l'obsession de la communication en Utopie au XVIII ${ }^{\mathrm{e}}$ siècle, et prépare à Bordeaux III une thèse en Communication sur le « Mécénat, outil de communication des entreprises : l'exemple des Pays de la Loire ». 